\title{
MATERIALS EXPOSURE AND DEGRADATION EXPERIMENT (MEDET)
}

\author{
M. Dinguirard* and J.C. Mandeville \\ ONERA, Toulouse, France \\ M. Van Eesbeek ${ }^{\ddagger}$ and A.P. Tighe ${ }^{\S}$ \\ ESA/ESTEC, The Netherlands \\ C. Durin', \\ CNES, Toulouse, France
}

\author{
A. Chambers, S. Gabriel ${ }^{\#}$, D. Goulty and G. Roberts \\ University of Southampton, Highfield, UK
}

\begin{abstract}
Material properties are of primary interest for spacecraft designers and their evolution as a consequence of exposure to the space environment must be accurately predicted. In this context, CNES, ESA, the University of Southampton and ONERA are participating in a cooperative effort to develop a test-bed called the Material Exposure and Degradation Experiment (MEDET). The experiment will be integrated on the European Technology Exposure Facility (EuTEF), and as such will be placed on the Columbus External Payload Facility, an exterior platform on the International Space Station. The objectives of the experiment are concerned with the effect of the complex space environment on the optical and thermo-optical properties of materials and the characterisation of the ISS environment. This paper describes the MEDET system configuration, the different scientific experiments, the control electronics and the overall implementation of the payload.
\end{abstract}

\section{INTRODUCTION}

ESA's early utilisation programme for the International Space Station provides the opportunity for payloads to perform scientific research and applications on external Space Station sites. The Materials Exposure and Degradation Experiment (MEDET) will fly in the framework of this programme as one of the payload modules integrated on the European Technology Exposure Facility (EUTEF). ${ }^{2}$ As such, the experiment will be located on the Columbus External Payload Facility, on the outside of ESA's Columbus Module.

MEDET is a co-operative effort between CNES, ESA, the University of Southampton and ONERA. It will consist of different sub-experiments contributed by the four parties and the sub-systems required to support them.

\footnotetext{
Head, Département Environnement Spatial

Département Environnement Spatial

* Section Head, Materials, Physics and Chemistry Section

$\S$ Research Fellow, Materials, Physics and Chemistry Section

II Centre Spatial de Toulouse

\# Senior Lecturer, Astronautics Research Group, School of Engineering Sciences

Copyright (C) 2001 The American Institute of Aeronautics and Astronautics Inc. All rights reserved.
}

The overall concept of the investigation is to actively monitor material degradation dynamics in Low Earth Orbit, and to characterise the local Space Station environment. After in-orbit operations are complete, MEDET will be returned to Earth so that the material samples can be analysed, and a comparison can be made between the in-orbit and post-operative results.

\section{Background}

The evolution of material properties in space is of primary interest for spacecraft designers and must be accurately predicted. In most cases the behaviour of such materials can be ground-tested using simulation facilities such as solar simulators, atomic oxygen sources, thermal cycling facilities and charged particle accelerators However, real space is difficult to simulate and synergistic effects on materials have been evidenced by past flight experiments. ${ }^{6,7}$

The ground simulation of the actual combined environments is generally quite complex. Due to constraints of a technical and financial nature, it has to be limited to the space environment parameters which are foreseen as the most active in actual flight conditions, according to the knowledge derived from sample return experiments. Therefore comparison of the degradation predicted in laboratory testing with that obtained in space is of the highest interest. It is 
also of great importance to know about the real effects of the degradation.

Past experiments, with degradation measurements only, have not always exhibited real in-space degradation effects as samples are often cured by oxygen in the air after their return. Moreover, the degradation dynamics, combined effects and saturation processes are not fully understood.

\section{Scientific Objectives}

MEDET has three main scientific objectives :

(a) Evaluation of the effects of the complex space environment on the optical and thermo-optical properties of materials to be considered for utilisation on LEO spacecraft. This includes the active measurement of thermo-optical properties (and more exactly, their variation as a function of space exposure) for a number of proposed materials. Among these are anodizations and other materials externally used on board LEO spacecraft (such as SSM and new thermal paints). Calorimetric devices will be employed for this purpose.

(b) Assessment of the effects of the ISS environment on optical windows. A special emphasis will be placed on the degradation process that is due to molecular contamination. Contamination results from various sources, such as out-gassing and degradation of materials, manoeuvres of the service vehicles, re-boost operations, firings of the attitude control systems, dumps and EVA. A spectrometer system will periodically measure the spectral optical transmission of transparent samples in the 0.2 to $1 \mu \mathrm{m}$ wavelength range.

(c) Investigation of micro-particles and debris fluxes (especially their variation as a function of time) and, after retrieval, the origin of the debris and the detectors' behaviour. Active capacitor type detectors (MOS) will be used to monitor the size distribution and the spatial-temporal variation of small dust particles.

In support of the above objectives, the local environment will be characterised as follows :

> Contamination will be monitored by QCM (Quartz Crystal Micro-balances).

$>$ A pressure gauge will check the ISS local pressure.

$>$ Atomic oxygen fluxes will be estimated by other QCM and thin film sensors.
$>\mathrm{X}$ ray and UV fluxes will be measured using photoelectric detectors.

If available, results from the other EuTEF or ISS monitoring devices will also be used to gain a better understanding of the effects of the plasma environment on the material properties under investigation.

\section{TECHNICAL DESCRIPTION}

\section{Accommodation on EuTEF}

MEDET will be accommodated on EuTEF as a standard payload, with dimensions of $548 \times 234 \times$ $219 \mathrm{~mm}$. It will require a free field of view towards the ram and zenith directions, and as such will be located in a corner on the lower tier of the Facility (see Figure 1). Power, command and communication interfaces will be provided on board by EuTEF.

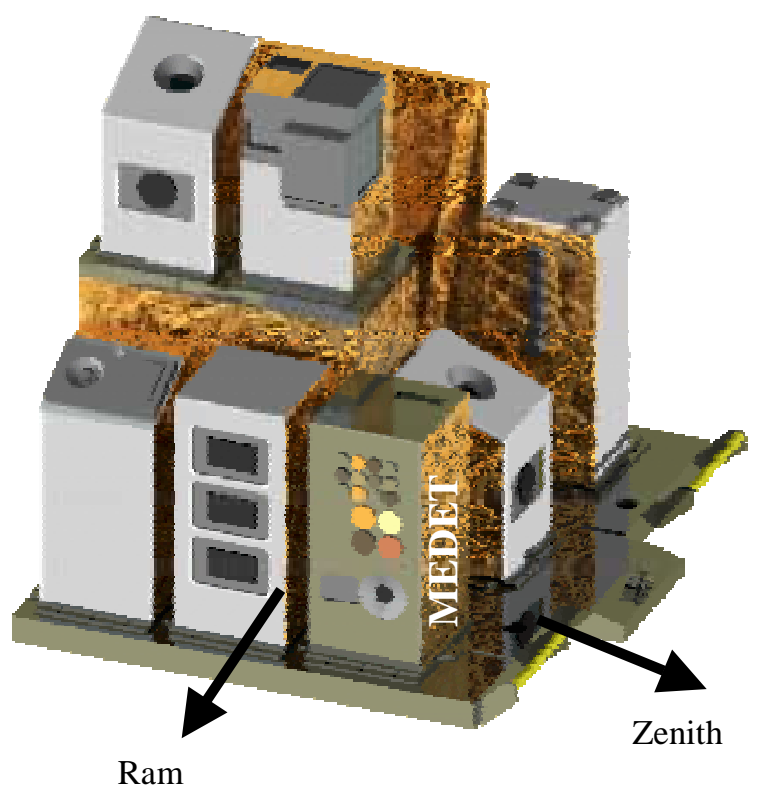

Figure 1 MEDET accommodation on EuTEF

\section{System Configuration}

MEDET will consist of a box-type aluminium support structure, to which the experiments and the electronics sub-system will be attached. The sensors and materials to be exposed will be located on the ram and zenith faces, and all other parts of the structure will be enclosed. Multi-layer insulation and radiators will be used on the outside of the structure for thermal control purposes. The overall layout of the system is shown in Figure 2. 


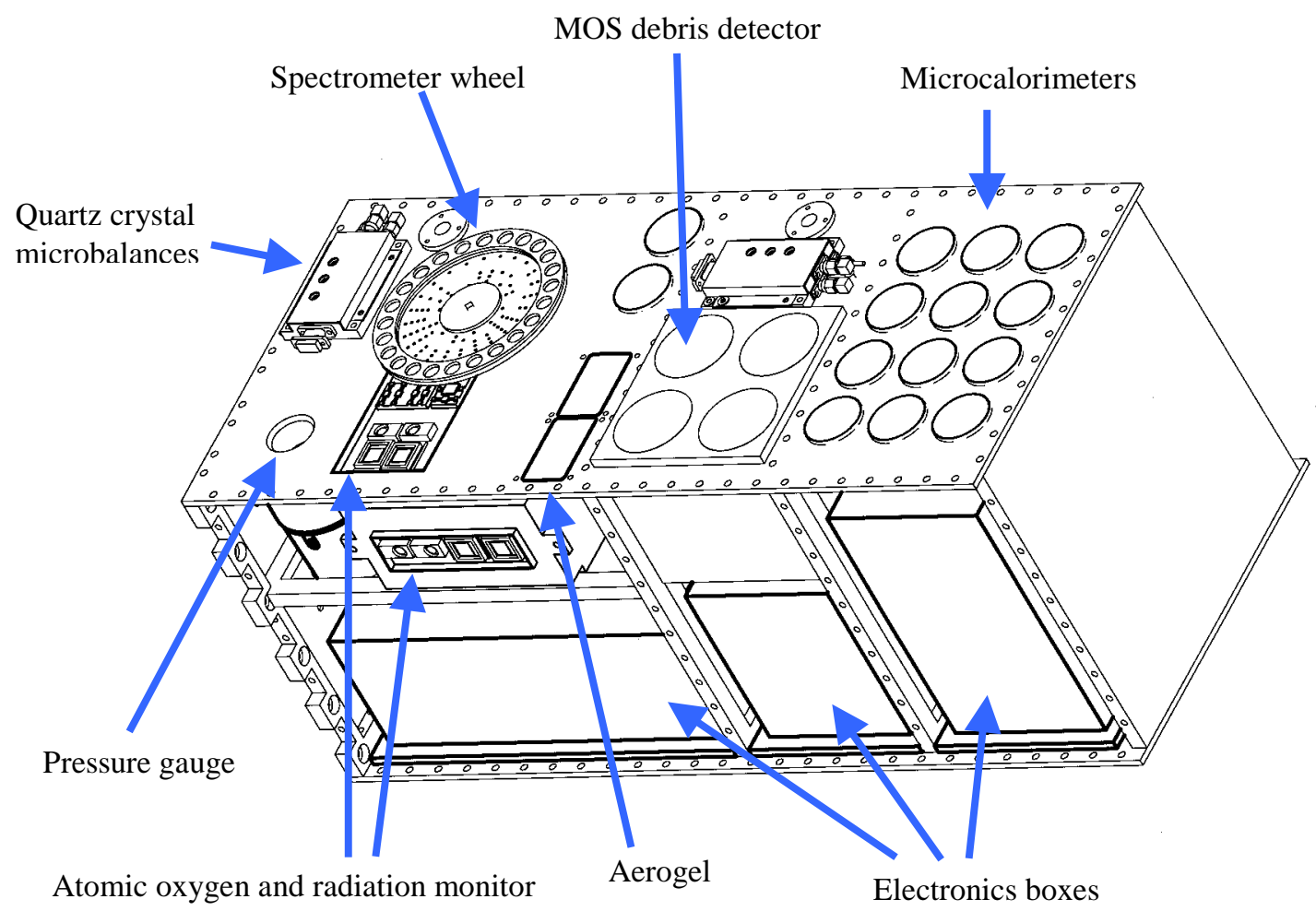

Figure 2 MEDET system configuration, showing ram and zenith face (zenith cover removed)

The electronics will consist of a central power unit to distribute power from the 28V EuTEF feed to each of the sub-experiments., and a software-driven central processing unit, to control the command and communication interfaces with each of the subexperiments. The scientific data will be transmitted to the MEDET ground segment periodically during onorbit operations, and it will also be possible to occasionally upload telemetry commands from the ground to adjust the operating parameters of the experiments.

Each experiment will operate and acquire data in different sequences and at different times, depending upon the scientific investigations it has to perform. For example, the spectrometer system will only acquire data when sunlight is incident on the ram face. The on-board software will be used to implement and monitor the processes.

\section{MEDET sub-experiments}

Seven sub-experiments will be integrated onto MEDET. Some of these experiments will contain material samples which will be exposed directly to the space environment, and some of the experiments will contain monitoring devices which will be used to actively measure the particle, molecular and electromagnetic fluxes impinging upon the samples.
The general concept of MEDET is to use as many already developed and space qualified systems as possible. Each of the sub-experiments is described in detail below.

\section{Micro-calorimeters}

Fourteen space qualified micro-calorimeters will be employed in order to follow the degradation of the thermo-optical properties of thermal control materials (TCC). Each calorimeter consists of a thermally insulated metal plate upon which the material to be tested is placed, using a technique such as vapour deposition, adhesive bonding or painting.

The temperature of the samples is measured using platinum resistance thermometers, and the equilibrium temperature variation for a given solar flux allows the variation in solar absorptance to be deduced. A black body calorimeter is used for reference purposes. The diameter of each calorimeter is $5.6 \mathrm{~cm}$, with a sample diameter of $2.5 \mathrm{~cm}$.

\section{Transmission Spectrometer}

This system is specially designed for MEDET's purpose in order to study the possible effects of contamination on the optical surfaces present on board the ISS and the degradation of optical windows as a consequence of the space exposure. 
Twenty-two transparent optical windows (including synthetic ultra-pure $\mathrm{SiO}_{2}$ and other radiation stable materials) will be placed on a rotating sample wheel on the ram face of MEDET. The sun will be used as a light source, and space resistant optical fibres will be used to transmit the light through the windows to miniature spectrometer modules.

Two holes in the wheel will remain empty in order to look directly at the Sun, for use as a reference source. An encoder system, consisting of photodiodes positioned under the wheel, will be used to identify the location of the wheel with respect to the fibre optic at any given instant during the measurement sequence (see Figure 3)

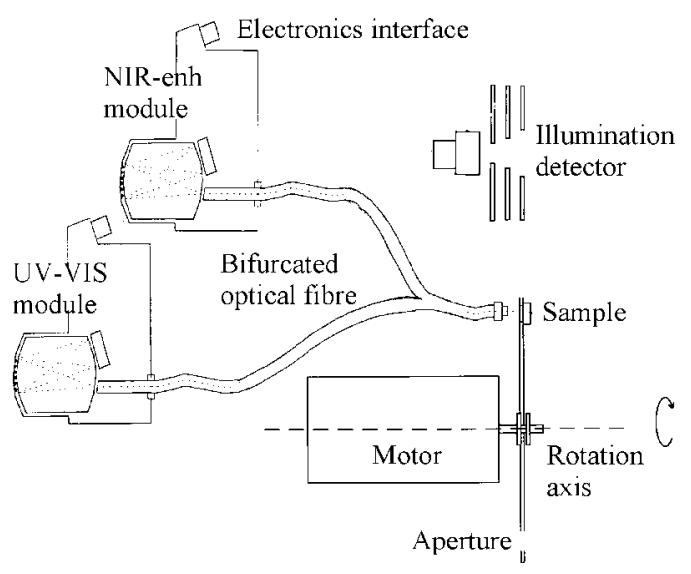

Figure 3 Schematic of optical system for transmission measurements

The spectrometer modules will be adapted versions of two commercially available devices, which are sensitive to wavelengths in the range 190-735nm (UV-VIS) and 350-1100nm (NIR-enh) respectively. Each module consists of a grating, a photodiode array detector, a fibre optic and associated electronics. The spectrometers and their fibres will be contained in a pressurised cylinder in order to avoid out-gassing. Two cylinders and two sets of spectrometers will be built for redundancy (see Figure 4).

The optical spectral transmission of the samples, in the wavelength range $200-1100 \mathrm{~nm}$, will be measured by looking at the Sun alternately through an empty hole and through each sample. Due to geometric considerations, the measurements will be allowed only when an optical sensor, having an acceptance angle of $\pm 40^{\circ}$, detects the sun.

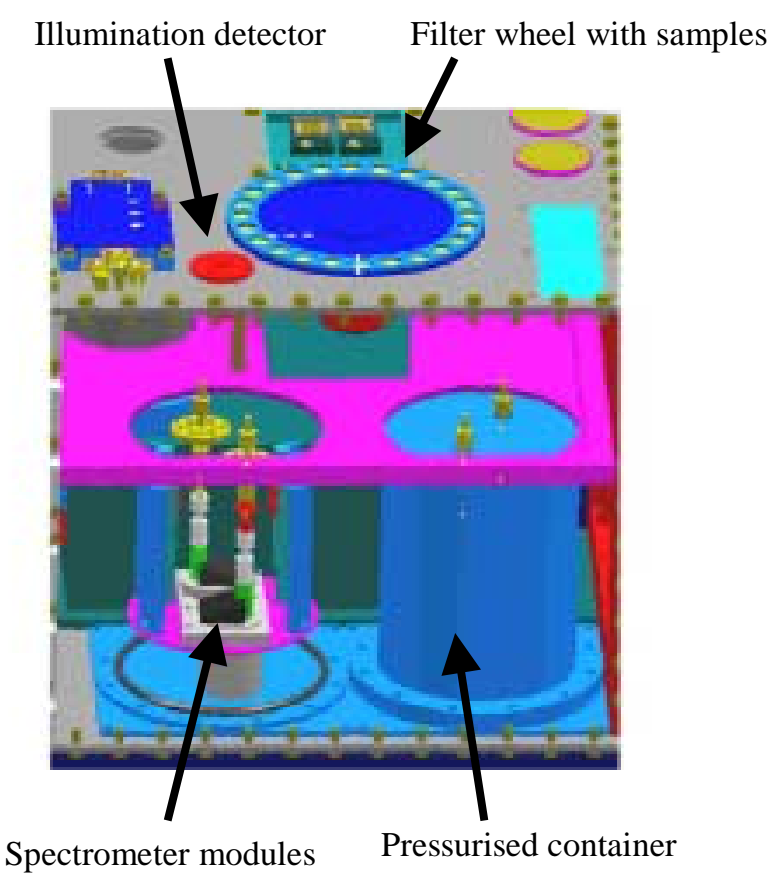

Figure 4 Spectrometer container and modules

\section{MOS detectors}

These sensors will be devoted to the active detection of micrometeoroids and debris. They are based upon the monitoring of the discharge of a parallel-plate capacitor using a thin dielectric. The device is operated with a bias voltage applied across the capacitor plates, so that a charge is normally stored in the capacitor. When a high velocity particle impacts the exposed plate with enough energy, it causes the dielectric to breakdown and the result is a discharge of the capacitor. The event is measured by monitoring the current required to recharge the capacitor. Evaporation of the electrode around the impact site usually prevents the occurrence of a permanent short. The sensitivity of the sensor depends mainly on the thickness of the top electrode and the dielectric.

The substrate of the detectors consists of p-type silicon wafers, 2 inches in diameter, polished on one side by mechanical and electro polishing. In order to form the capacitor, a layer of oxide is grown on one face of the wafer by thermal oxidation. A thin layer of aluminium is then vapour deposited on each face to form the top electrode and electrical contact to the silicon wafer. The thickness of the dielectric is 1.4 $\mu \mathrm{m}$ and the thickness of the Al top electrode is 0.1 $\mu \mathrm{m}$ (see Figure 5). Prototypes have been tested with micro-particle accelerators. The device is best suited to the detection of particles with diameters ranging from $0.5 \mu \mathrm{m}$ to $100 \mu \mathrm{m}$ and for an impact velocity larger than $2.5 \mathrm{~km} / \mathrm{s}$. 


\section{$\downarrow \downarrow \downarrow \downarrow \downarrow \downarrow$}

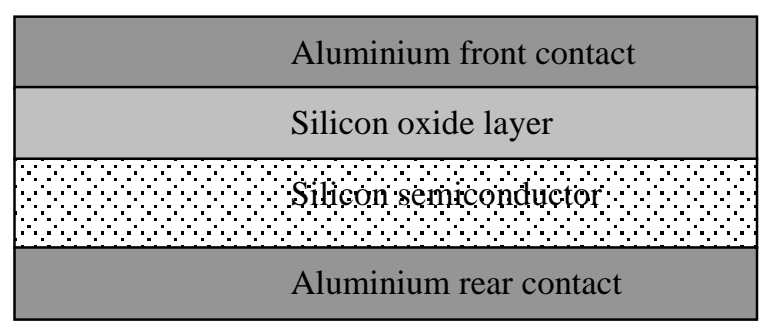

Figure 5 Cross section of a MOS sensor

A module made of 4 MOS detectors, already developed for a Franco-Brazilian mini-satellite (FBM), will be used in MEDET (see Figure 6 ). The module will require $28 \mathrm{~V}$ power, command and data handling interfaces. ${ }^{1}$ Retrieval after exposure to space will allow a comparison of in-orbit detection data with actual impact features found on the device. This appraisal will be valuable for future improvement of detectors.

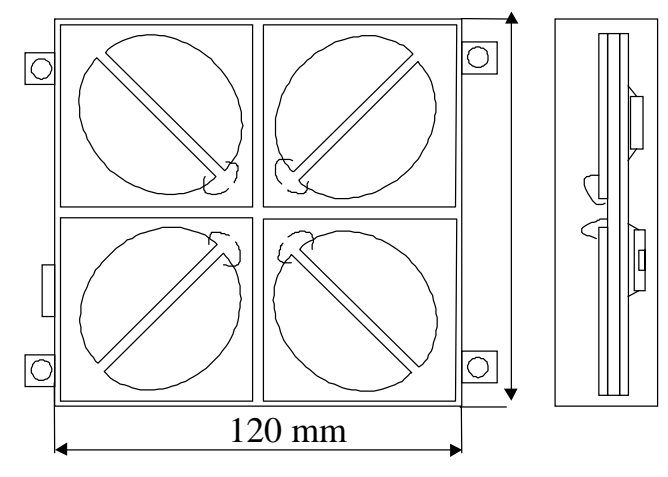

Figure 6 Schematic of the MEDET module of four MOS detectors

\section{Aerogel}

A block of aerogel will be placed on the ram face of MEDET to capture micrometeoroid and debris particles. Aerogel is an ultra-low density glass (typical density $0.1 \mathrm{gcm}^{-3}$ ), which can decelerate high velocity particles without totally vaporising them. The experiment will be passive, and the aerogel will only be analysed once MEDET has been returned to the ground and de-integrated.

The chemical composition of the particulate residue left in the aerogel will be determined in order to differentiate between debris and micrometeoroids, and the angle and length of the tracks will also provide information about the original impact angle and velocity of the particles.

\section{Pressure Gauge}

An adapted commercial cold cathode type pressure gauge will be used to measure the local pressure. The characteristics of the instrument will be as follows :

Pressure measurement range : $10^{-2}$ to $10^{-9} \mathrm{mbar}$ Power consumption : $2 \mathrm{~W}$

Signal output : 0-10V with $2 \mathrm{mV}$ resolution

The data from this experiment will also be collected by the central EuTEF Data and Power Handling Unit, and made available to the other investigations on board the Facility.

\section{$\underline{\text { QCM experiment }}$}

The Quartz Crystal Microbalance (QCM) experiment will be used to measure contamination levels and the atomic oxygen flux in the vicinity of the Space Station. The sensors will be modified versions of commercially available miniature crystal oscillators. The quartz crystal is exposed to the space environment through a round hole in the housing directly above the crystal, and the oscillation frequency changes in relation to the changing mass and temperature of the crystal. A similar concept has been used for devices flown on board ESA's Artemis satellite. ${ }^{8}$

To measure the contamination flux, a bare crystal is used, and the oscillation frequency decreases as contamination is deposited on the surface of the crystal and the mass of the crystal increases. To measure the atomic oxygen flux, a carbon coated crystal is used, and the oscillation frequency increases as the atomic oxygen erodes away the carbon layer and the mass of the crystal decreases. For both of these techniques, the relationship between the mass of the crystal and the oscillation frequency can depend on the temperature of the crystal. Therefore, a separate non coated crystal cut in the "Y-direction" is also used to independently monitor the crystal temperature, so that the mass data can be corrected for temperature dependent effects.

Three types of sensor will be flown on board MEDET :

- $\quad$ Type 1 : $10 \mathrm{MHz}$ Crystal with Au electrode for contamination deposition measurements. The sensitivity is $4.4 \times 10^{-9} \mathrm{gcm}^{-2} \mathrm{~Hz}^{-1}$.

- Type 2 : $10 \mathrm{MHz}$ Crystal with C-coating, to measure Atomic Oxygen. The sensitivity is $2.46 \times 10^{15} \mathrm{O}$-atoms $\mathrm{cm}^{-2} \mathrm{~Hz}^{-1}$. 
- $\quad$ Type 3 : $11 \mathrm{MHz}$ Crystal with Au electrode, for temperature measurements. The sensitivity is 600 $\mathrm{Hz} / \mathrm{K}$. The output of this type of crystal will be used to correct for temperature variations in Type 1 and Type 2.

The response of each type of crystal is only linear within a limited frequency range. If a sudden large contamination deposit were to occur, perhaps due to a thruster firing in the local vicinity of MEDET, then the experiment would no longer be operable. Therefore, a heater and thermostat will also be placed underneath each crystal, so that an attempt could be made to "de-contaminate" the sensors by evaporating off the excess deposits.

The three sensors, the heaters and the associated electronics will be contained within an aluminium box (see Figure 7) and two such boxes will be placed on the ram face of MEDET.

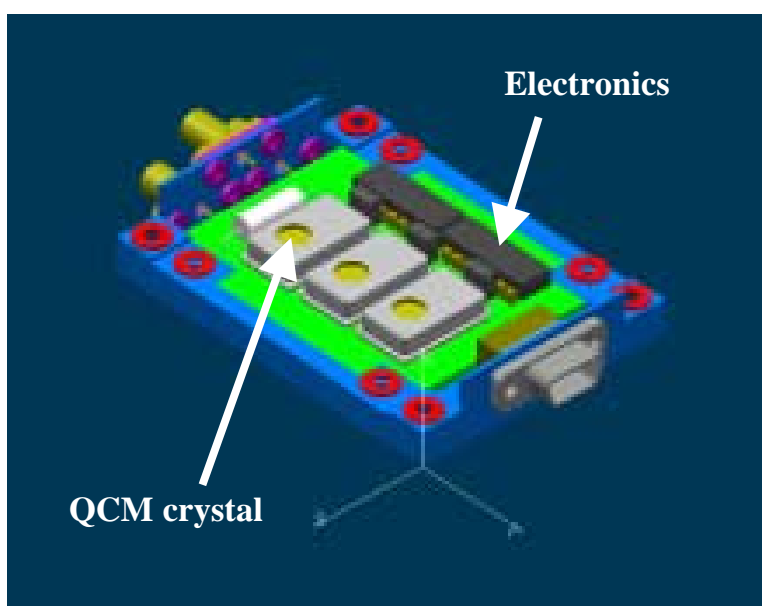

Figure 7 QCM experiment (upper cover not shown)

\section{$\underline{\text { Atomic oxygen and radiation monitor }}$}

A package of sensors developed by the University of Southampton will be used to monitor Atomic Oxygen, UV and X-ray fluxes.

Two AO sensor types are to be incorporated, based on carbon film actinometers and thin-film zinc oxide $(\mathrm{ZnO})$ detectors. Both will be located on the ram-face of MEDET in an area close to the rotating spectrometer wheel. Both types of AO sensors use the thin film resistance as the measurand.

For the carbon film actinometers, the film resistance is expected to increase progressively as the carbon is eroded/oxidised by the AO. The principle of operation is similar to that of silver-film actinometers previously designed by the University of Southampton and flown on the STRV-1a mission. ${ }^{3}$

The carbon actinometer instrument will comprise four thin carbon films and an AD590 temperature sensor, all mounted on an alumina substrate the dimensions of which are $17 \mathrm{~mm} \times 36 \mathrm{~mm} \times 0.635 \mathrm{~mm}$.

Incorporated onto the substrate will be a low power heater element that will be used to stabilise the film temperature since carbon exhibits a temperature dependent erosion rate.

Zinc oxide is an n-type semiconductor material that is sensitive to adsorbed atomic oxygen. Analysis of the non-steady response indicates that the initial rate of decrease of the conductivity of a fresh $\mathrm{ZnO}$ film exposed to atomic oxygen is proportional to the incident AO flux. ${ }^{5}$ However, after a relatively short exposure to $\mathrm{AO}$ the sensor becomes saturated and its response ceases. Nevertheless, the sensor offers the great advantage that, by heating to modest temperatures, the sensor can be refreshed (or "regenerated") and is then able to take further AO measurements.

The $\mathrm{ZnO}$ sensor will comprise four thin films, two of which will be shielded from AO attack by a thin overcoat of silicon monoxide (silica). As the $\mathrm{ZnO}$ films are also responsive to UV, the silica-coated sensors will form a control experiment by which the effects of UV and AO can be separated. As with the carbon actinometers, the films will be deposited onto an alumina substrate onto which a low power heater element will also be deposited. The heater element is designed to stabilise the film temperature in the long term and to provide a heating pulse for regeneration purposes. The latter will be achieved via capacitative discharge. An AD590 temperature sensor will be used for steady-state temperature measurements.

The carbon actinometer elements will be fully exposed on the ram face, whereas the $\mathrm{ZnO}$ film array will be partially covered by the spectrometer wheel. One sensor is permanently shadowed from direct exposure to AO by the wheel, whilst another is periodically covered/uncovered by the wheel. The other two sensors are fully exposed on the ram face. $\mathrm{ZnO}$ sensor regeneration will take place whilst two of the four sensors are covered by the wheel.

Four x-ray sensors will be mounted on MEDET. Two will be placed on the zenith face and two placed on the ram face with one active and one redundant active sensor on each of the faces. The x-ray sensors will consist of silicon PIN diodes. These will have a thickness of $500 \mu \mathrm{m}$ and an active area of $100 \mathrm{~mm}^{2}$. A $25 \mu \mathrm{m}$ layer of beryllium will be placed over the 
diodes to filter out the signal from lower energy photons. The low band pass filter will be supplied by the thickness of silicon sensor itself. The sensors' mass with their associated electronics will be of the order of 5 grams each. Power consumption will be approximately $200 \mathrm{~mW}$ per sensor. The energy range of the detectors will be from $1 \mathrm{keV}$ to $10 \mathrm{keV}$. X-ray fluxes will be measured continuously, but the most important data will be gathered during Solar x-ray flare events when the x-ray flux is at its highest and most damaging levels. The expected flux to the detectors will be between $1 \mathrm{pW}$ to $10 \mathrm{nW}$, this energy range covering four orders of magnitude from typical quiet solar activity to $\mathrm{X}$-class flares.

The detectors will operate in the photovoltaic mode to cut down on dark current, a necessary precaution considering the tiny signal power. The output current from the detectors will be passed to a transimpedance amplifier array that will produce two output signals of $0-10 \mathrm{~V}$, one output to cover the range from $1 \mathrm{pW}$ to $100 \mathrm{pW}$, the other to cover $100 \mathrm{pW}$ to $10 \mathrm{nW}$. Recording frequency will be one measurement from each of the four sensors every 30 seconds. Each sensor will have an attached PT100 sensor to monitor the temperature during each recording so the output signal can be calibrated against temperature sensitivity.

Eight UV sensors will be mounted on MEDET, four on the ram face and four on the zenith. The UV sensors will consist of solar-visible blind aluminium gallium nitride photodetectors. These detectors are sensitive to an UV light range from $120 \mathrm{~nm}$ to $285 \mathrm{~nm}$, and this lower limit of the wavelength range is important to be able to detect the Lyman-alpha hydrogen line emitted at $121.6 \mathrm{~nm}$ ( which is the strongest line in that region of the spectrum). To further enhance the performance of Lyman-alpha detection, half the sensors (two on each face) will be fitted with sapphire windows to cut out the UV spectrum below approximately $130 \mathrm{~nm}$. Deduction of the filtered sensor signal from the bare sensor signal will produce a measurement of the $120-130 \mathrm{~nm}$ region that will be dominated by changes in the Lymanalpha flux.

The detectors have a diameter of $250 \mu \mathrm{m}$ and are mounted in a standard TO5 can. Future testing will also be required to monitor the erosion rates and changes in performance of the detectors when exposed to atomic oxygen and electron fluxes. The mass of each sensor and the associated electronics will be approximately $4 \mathrm{~g}$. Power consumption of each detector will be approximately $100 \mathrm{~mW}$. The expected flux to each detector will be approximately $650 \mathrm{nW}$ and the output current generated will be passed to a transimpedance amplifier for conversion to a $0-10 \mathrm{~V}$ output signal. The recording frequency will be one measurement for each sensor every five minutes. There will be six PT100 sensors, three on each face, to monitor the temperature during each recording so the output signal can be compensated for temperature sensitivity.

\section{Electronics sub-system}

\section{Power supply}

MEDET will use its own power supply to convert and distribute the power from the $28 \mathrm{~V}$ EuTEF feed to each of the sub-experiments and the logic unit. For reasons of safety and noise immunity, he subexperiments will be divided into groups, and a DC/DC converter will be used to power each group separately. Current limiters and switches will be used to isolate the experiments in the event of an abnormally high current reading.

\section{Logic unit}

The central logic will be used to command and control the operation of the sub-experiments, the acquisition and initial storage of the data and the interfacing with EuTEF. The core of the logic unit will be arranged into two FPGAs (Field Programmable Gate Array). One of these devices will control the high level management of the system, such as telecommand interpretation and fault finding. The other will carry out low level tasks associated with the commanding of the sub-experiments. A schematic of the logic unit, the power supply and the command and communication interfaces is shown in Figure 8.

\section{IMPLEMENTATION}

\section{Qualification}

The qualification of MEDET will be based on a protoflight approach. A functional model will be manufactured and tested, followed by one prototype model.

\section{Functional Model}

The purpose of the functional model is to ensure that all of the electrical and electronic systems will function correctly as an integrated system, to ensure that MEDET will communicate correctly with EUTEF, and to test and qualify the flight software.

The functional model will consist of breadboards of the logic unit, power supply and electronics interfaces, flight control software, either breadboard models or engineering models of the sub-experiments and a mock-up of the MEDET - EUTEF interface. 


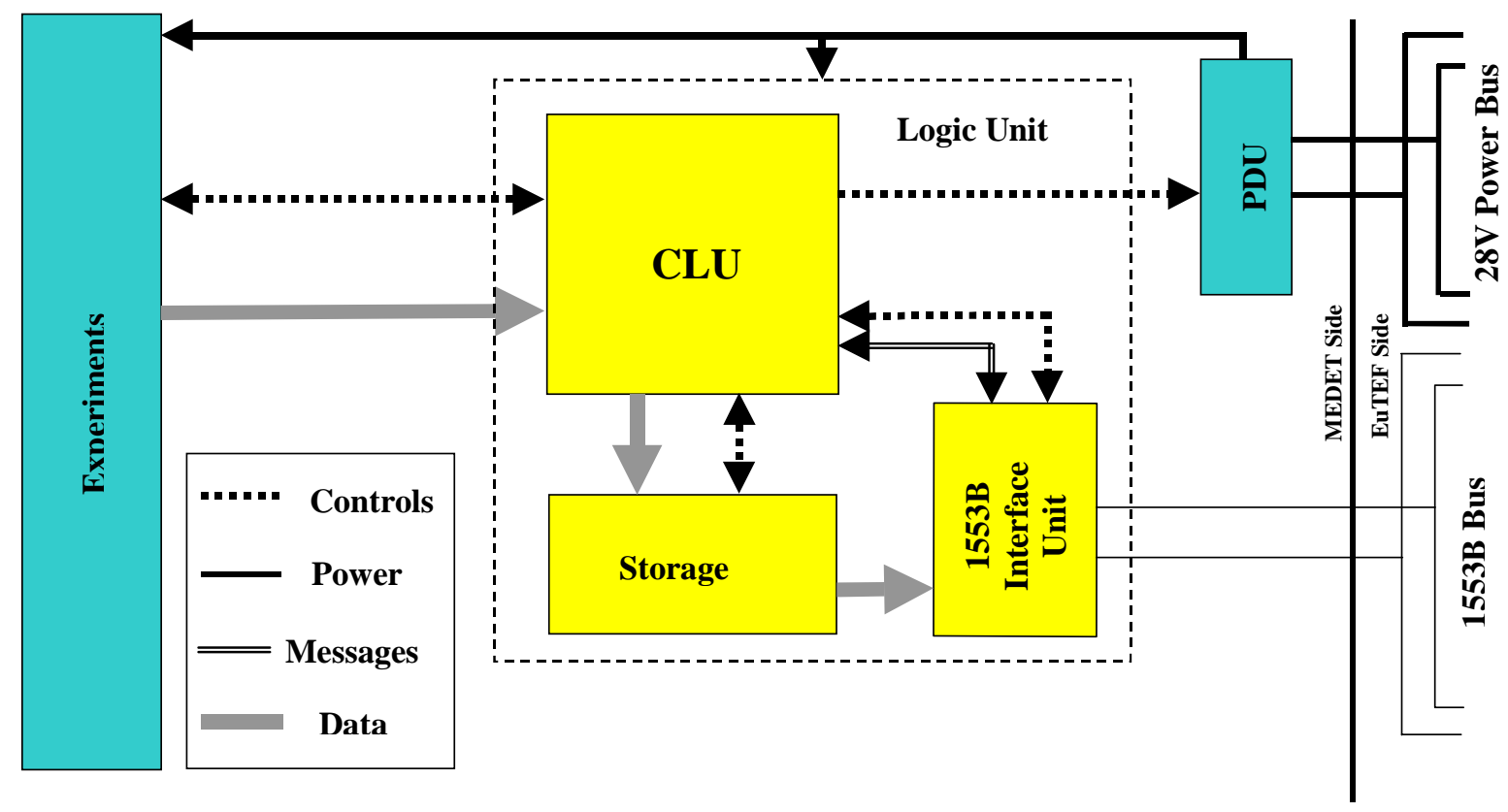

Figure 8 MEDET electronic sub-system architecture

\section{Protoflight Model}

The protoflight model is the final system which will be integrated onto EuTEF A series of environmental and verification tests will be carried out on this model, to demonstrate the compliance of the system with all of the applicable requirements.

\section{Transport and Storage}

During transportation and storage phases, MEDET will be kept under controlled environmental conditions, and sensitive surfaces will be protected using covers or housing racks. These will be removed before launch. In addition, the samples and sensors will be integrated as late in the development cycle as possible. This will minimise degradation and contamination on the ground, and will allow the most up to date materials and coatings to be considered for the investigation.

\section{Operations}

When in orbit, the experiments will be nominally exposed to space for three years. Regular data will be sent to ground via the EuTEF and ISS data links. At the end of mission, MEDET, as part of EuTEF, will be retrieved and returned to earth for laboratory investigation. EuTEF is due for launch on the US Space Shuttle in early 2005.

\section{CONCLUSION}

The development of a new space flight experiment which will carry out materials exposure and degradation studies in the frame of the Space Station early utilisation program for external payloads has been described. A collection of sensors will allow active measurements to be carried out in orbit, before the experiment is returned to Earth for analysis.

The environment sensors associated with the microcalorimeters and the transmission spectrometer will permit differentiation of the effects of the various components of the space environment, including those of contamination and AO. This will also allow further study of damage kinetics in actual space conditions.

Comparison between laboratory simulation test data and the in-flight data will enable one to validate and to improve the ground testing procedures. The MOS module and aerogel experiment will also allow more information to be obtained on the micro-particle fluxes present in the ISS orbit.

All these study results can be obtained due to the unique advantages of the EuTEF, including experiment modularity, power supply and data links. The various experiments and sub-systems are now fully designed and MEDET is in the C/D phase. 


\section{ACKNOWLEDGEMENTS}

Figure 1 has been included courtesy of ESA and CGS and Figure 8 has been included courtesy of TRECE. Figures 2, 4 and 7 were produced by the MEDET mechanical design team at ESA.

The development team at the University of Southampton would also like to acknowledge the generous assistance of Professor Neil Ross from the Department of Electronics and Computer Science for the design of the electronic systems for the AO, X-ray and UV instruments.

\section{REFERENCES}

1. Durin D. and Mandeville J.C., "MOS sensors for the detection of micrometeoroids and space debris", Second Conference on Space Debris, ESA, ESA SP-393.

2. Dettmann, J. and Borghi G., "The European Technology Exposure Facility (EuTEF) Developement and Adaptation", these proceedings

3. Harris I.L., Chambers A.R. and Roberts G.T., "A low cost micro-satellite experiment for the in-situ measurement of orbital atomic oxygen", Review of Scientific Instruments, vol. 68, no. 8, August 1997, pp. 3220-3228.

4. Mandeville J.C., "Impact detection in space: derivation of physical properties of meteoroids and debris", Adv. Space Sci., 17(12), pp. 147$153,1996$.

5. Osborne J.J., Roberts G.T., Chambers A.R. and Gabriel S.B..,"Thin-film semi-conductor sensors for hyperthermal oxygen atoms", Sensors and Actuators B, Vol. 63, no. 1-2, April 2000, pp. 5562.

6. Silverman E.M., "Space Environmental Effects on Spacecraft: LEO Materials Selection Guide", NASA Contractor Report 4661, NASA Langley Research Center, August 1995.

7. Whitaker A.F. and Gregory J., "LDEF Materials Results for Spacecraft Applications", Proceedings of a Conference held at Huntsville Alabama, Oct. 27-28, 1992, NASA Conference Publication 3257.

8. Viddeleer, R., "A Miniature Active Contamination Sensor", Preparing for the Future, ESA, vol 6., no. 1, March 1996 